\title{
Elementary Laboratory Assays as Biomarkers of Ageing: Support for Treatment of COVID-19?
}

\author{
Thomas Lung $^{\mathrm{a}}$ Pasquale Di Cesare ${ }^{\mathrm{b}}$ Lorenz Risch $^{\mathrm{a}} \quad$ Urs Nydegger $^{\mathrm{a}}$ \\ Martin Risch ${ }^{c}$

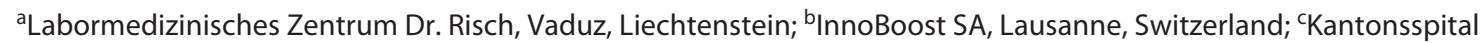 \\ Graubünden, Chur, Switzerland
}

\section{Keywords}

Biomarker · Senescence · Life expectancy · Ageing · Medical laboratory

\begin{abstract}
Youth, working age and the elderly: On a timeline, chronological age (CA) and biological age (BA) may dissociate; nosological entities manifest themselves at different BAs. In determining which disease corresponds to a given age decade, statistical registries of causes of death are unreliable and this does not change with SARS CoV-2 infection. Beyond adolescence, ageing metrics involve estimations of changes in fitness, including prediction models to estimate the number of remaining years left to live. A substantial disparity in biomarker levels and health status of ageing can be observed: the difference in CA and BA in the large cohorts under consideration is glaring. Here, we focus more closely on ageing and senescence metrics in order to make information available for risk analysis non the least with COVID-19, including the most recent risk factors of $A B O$ blood type and 3p21.31 chromosome cluster impacting on C5a and SC5b-9 plasma levels. From the multitude of routine medical laboratory assays, a potentially meaningful set of assays aimed to best
\end{abstract}

reflect the stage of individual senescence; hence risk factors the observational prospective SENIORLABOR study of 1,467 healthy elderly performed since 2009 and similar approaches since 1958 can be instantiated as a network to combine a set of elementary laboratory assays quantifying senescence.

(c) 2021 S. Karger AG, Basel

\section{Introduction}

The predominance of COVID-19 in the elderly, especially in those suffering from comorbidity, explains itself with immunosenescence and inflammaging [1]. The global burden of diseases might be studied in disciplines once thought to be completely separated, from paediatrics to geriatrics. Indeed, a kind of continuum seems to exist between growth and ageing [2], with the latter driven by increasing cellular and systemic function growthpromoting pathways after growth is completed (hyperfunction theory). This view of ageing and senescence impacts the definition of "disease" as, while still unnoticed, it can be possibly measured before its clinical manifestations in its pre and pre-pre-disease stages [3-5]. A preprint about an universal DNA methylation (DNAm) 
across mammalian tissues [6] reinforces the association and continuity between aging and development hinting to a conserved, gradual evolutionary process. Understanding that age is a major factor for attack rate of COVID-19 may help to design approaches against this disease that target the aging process [7]. Medical laboratory tools and biomarkers used to distinguish biological from chronological age (CA) have made substantial advances. This distinction manifests itself in the healthy development from young ages and already dissociates biology from chronology [8]. Laboratory care check-ups in healthy women and men allow for risk analyses for diseases and allow individuals take prophylactic measures to delay or prevent impending disease. Such programs my include analysis of the complement system when it comes to new diseases like COVID-19 [2, 9, 10].

The concept of studying the molecular mechanisms underlying the age-related decline of the organism, for example, cellular senescence, rather than the genetics of single disorders, is now performed by concurrently testing multiple susceptibility variants in combination with traditional risk factors [11]. The deferred disability adjusted life years (DALY) status of an individual might be subject to risk analysis supported not only by advanced and complex laboratory investigations with blood.

In Switzerland in the year 2017, 434,000 people were over 80 years of age and 77,000 over 90 years of age, and the country now hosts close to 2,000 centenarians. Motivated to better understand this senior population, its relative blood biomarkers and their reference ranges, we started an observational, prospective cohort study on 1,467 healthy uniracial Caucasian residents $>60$ years of age (SENIORLAB) back in the year 2009 [12]. One hundred five frequently used laboratory tests were performed for one blood sample. With a non-restrictive definition of health, several pathological laboratory results pointing to occult disease have been found and published $[12,13]$.

Classification by nosological denomination of particular diseases requires periodical updates because of the ageing of the population [14]. Organismal senescence at the organ level will become stageable and classified like any disease listed according to ihmeuw.org/4pxn or ICD$11[15,16]$. Machine-learning-based predictors of biological age (BA) at the organismal level and population level have recently been developed [17].

Artificial intelligence is likely to transform the way we look at diseases, their classification and link to ageing [17, 18]. Expert committees often classify diseases under neighbouring specialties of medicine, such as stroke, which bridges neurological disease and angiology or hae-

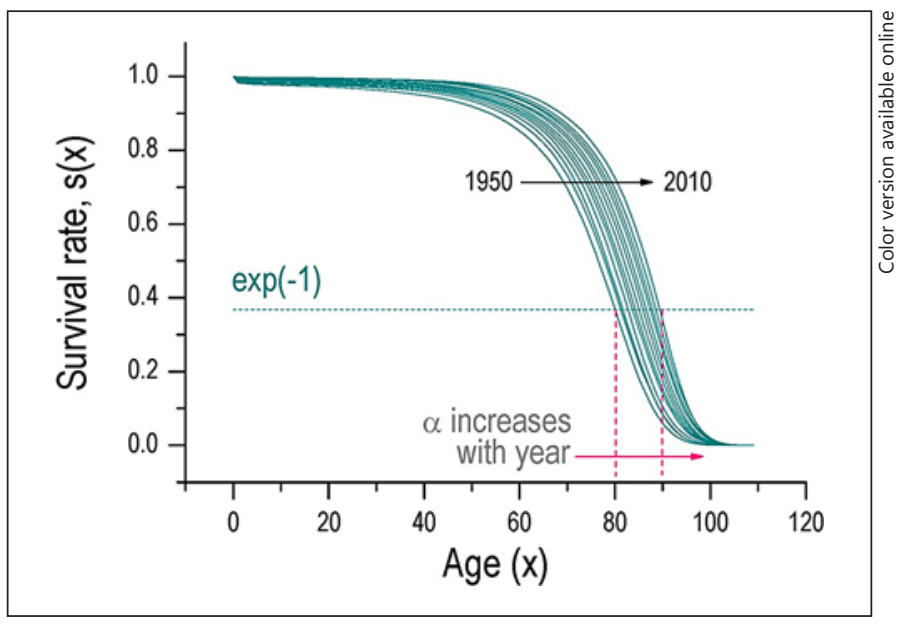

Fig. 1. Scale and shape of survival curves for Swedish females. Curves drawn from past 6 decades (1950-2010; interval 5 years) are used. A gradual shift rightwards and towards a rectangular shape becomes apparent. The characteristic life ( $\alpha$ ) for each survival curve was accurately measured by detecting the interception point between the $s(x)$ (solid line) and $s(\alpha)=\exp (-1)$ dashed line. The scale effect, revealed by the $\alpha$ value, can be removed by rescaling the age with $U=x / \alpha$, and allows quantification of the extent to which survival curves become rectangular as populations become older; from [26], with permission.

mostaseology, an aspect on the agenda for the 2018 formulation of ICD-11. A proposal currently exists for ICD11 to include an extension code (XT9T) for "age-related diseases"; thus, XT9T defines age-related diseases as those "caused by pathological processes that persistently lead to the loss of organism's adaptation and progress in older ages" $[17,19]$. As yet, the matter is subject to further appraisal [20].

\section{Physiology of Senescence}

The physiology of senescence related to simple routine med laboratory analyses in combination with other clinical results, when defined as the changeless irreversible cessation of cell division capacity (proliferative arrest) of a given cell type $[21,22]$, acquires a risk-factor stigma for age-related diseases. The difference between benign maturational changes and the senescence-associated pre-pre disease syndrome has been discussed previously $[23,24]$.

Differences in the causes of death among species and among different human races and different individuals is mirrored by survivor curves [25]. Demographic life tables allow one draw survival curves that reveal changes in scale and shape during the last few decades of life. The causes 
Table 1. Delineation of Senescence by Markers increased in the Elderly; a selection of cell types and their endurance during senescence is listed

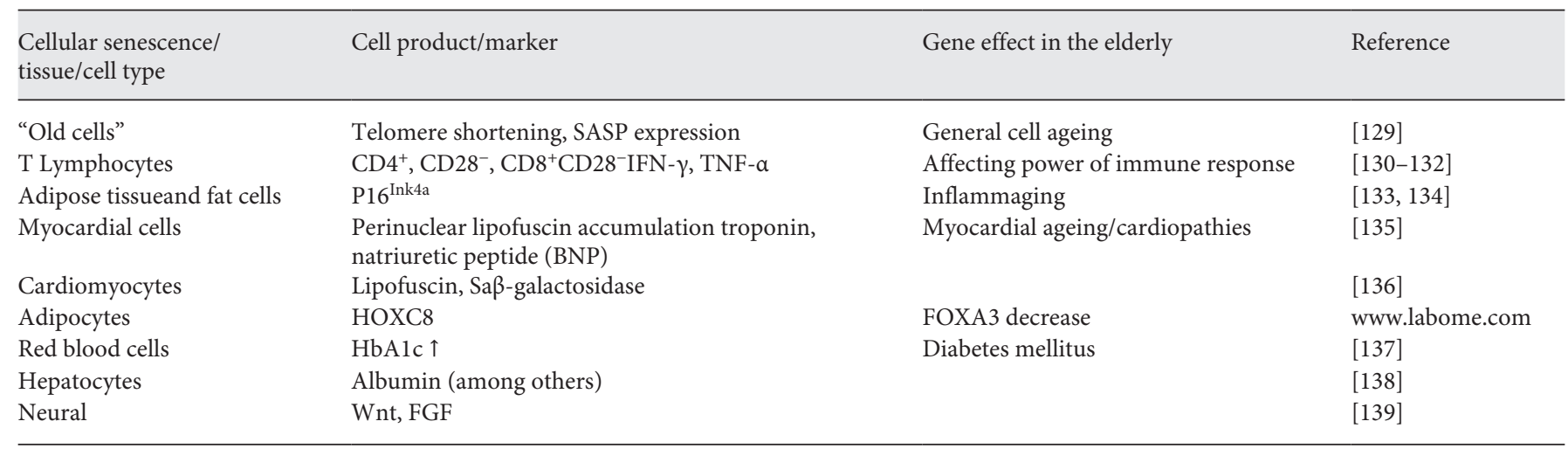

SASP, senescence-associated secretory phenotype; CD, cluster of differentiation; IFN, interferon; P in P16, protein; HOXC, homeobox C cluster; SAHFS, senescence associated heterochromatin focus; FOXA, Forkhead Box; H2A, Histone H2A, a component of DNA; HbA1c, glycated haemoglobin.

for this phenomenon are discussed below. A shift to the right acquiring a rectangular shape of the curve can be seen in Figure 1, reflecting the prolongation of life expectancy, as published recently [26]. One cannot exclude that a minority of the fittest, that is, the centenarians, shifted the curve, which would result in a survival bias. However, biomarkers of age will likely allow us to sort out such cases. In particular, DNAm clocks have broad applicability, but tissue-specific and multi-tissue age prediction cannot currently be distinguished [27]. Such a shift is adamant to simple med laboratory tests as well.

Cellular senescence imposes permanent proliferative arrest on cells in response to stressors, an event easier to study in cultured cells and less understood in situ of living organisms [7]. It is likely that the technical limitations relating to the identification of senescent cells in tissues and organs will soon be overcome. At least, beneficial signalling functions of senescence modulate the secretome, which is currently an area of research focus for anti-ageing therapy, as shown below [28]. Senescent cells loose functional components of earlier life but also acquire new activities: a pro-inflammatory paracrine effect on surrounding cells will impact tissue function, especially when the senescence-associated secretory phenotype (SASP) becomes identifiable (Table 1). Cellular senescence is a potent anticancer mechanism that arrests the proliferation of mitotically competent cells, preventing malignant transformation. Many proteins produced by cells that are involved in the process of cell signalling and immune function in ageing and regeneration can now be assessed by cytokine secretion profiles using techniques with surfaces of beads coated with analyte-specific cap-

Laboratory Assays as Biomarkers of Ageing ture antibodies (e.g., QBeads MultiCyt ${ }^{\mathrm{TM}}$ ). Multiplexed analysis of senescent cells has shed light on cell activation extents. Cellular senescence triggers the activity of mammalian target of rapamycin (mTOR), which can decrease the production of SASP through inhibition of mTORC1 [29]. Restriction of dietary branched-chain amino acids prolongs life span in mice [30]. A multinational study under the auspices of Novartis recently was initiated to assess the effects of mTOR inhibition on human ageingrelated conditions. In elderly volunteers, the response to influenza vaccination was tested using the mTOR inhibitor RAD001 to examine its ability to delay immunosenescence. The secrecy coded RAD001 substance enhanced the response to influenza vaccine by approximately $20 \%$ at doses that were relatively well tolerated. RAD001 also reduced the percentage of CD4 and CD8 T lymphocytes expressing the programmed death-1 receptor, which is now acknowledged to inhibit $\mathrm{T}$ cell signalling. Programmed death -1 is highly expressed in association with age. Thus rapamycin and other rapalogs (i.e., everolimus and temsirolimus) are under investigation to defend patients against SARS-CoV-2 infection [31]. These results raise the possibility that mTOR inhibition may have beneficial effects on immunosenescence in the elderly [32].

\section{Markers for Senescent Cells}

To denominate a cell as a senescent cell, one needs to define the functional properties or markers that have been lost or, conversely, acquired. Cell senescence induces interleukin release [33] and identification of senescence-associated $\beta$-galactosidase ( $\mathrm{Sa} \beta$-gal) activity in tissues, detectable at $\mathrm{pH}$ 6.0, which is now known as a marker for 
senescent cells [34]. Thus, such cells can be identified in tissues, and the original findings by Storer et al. [35] still require confirmation. Unexpectedly, using mouse embryos cultured on fertilized hen's eggs, a programmed component of embryonic development was identified [35]. The caveats of examining $\mathrm{Sa} \beta$-gal as an axiomatic marker for senescent cells have been outlined elsewhere [36]. Markers for senescence of senescent cells are manifold: the right constellation to denominate a cell as senescent is quite solid but not unequivocal [37]. The daily pattern of laboratory tests in a doctors' office counts few analyses, for example, creatinine, $\mathrm{HbAlc}$, or ferritin among others.

The myriad of different surface structures on cells, that is, surface proteins and surface glycosaminoglycans, can be used for cell allotment, typing or to distinguish healthy from diseased tissues. In oncology and haematology with the ease of accessing (leukaemic) cells, progress in proteome characterization is evident. The common set of proteins used to deploy oncogenic perturbations in B cells, such as FLT3, NCR3LG1 or ROR1, may be profiled using genetically barcoded antibodies [38].

Cell senescence is sometimes defined as the irreversible cessation of cell division capacity (proliferative arrest) of a given cell type [28]. Human cellular senescence genes are accessible in databases (https://genomics.senescence.info/cells/), and a genetic background for cellular senescence does not appear to be controversial [37].

Apoptosis, which was originally regarded as an isolated event that does not affect surrounding tissue, is now perceived as a response to stress and injury that causes the dying cell to secrete, upon death, mitogenic and morphogenic substances that stimulate growth and repair in their surroundings. A cohort of dying (senescing?) cells must then be observed during normal development and in pathological conditions [39].

Cancer and its treatment consist of stress factor-accelerating senescence. Modern treatment protocols, such as chimeric antigen receptor bearing $\mathrm{T}$ lymphocytes or combined cytostatic regimens, as well as early detection and supportive care, have resulted in an estimated 16 million cancer survivors who are alive in the United States [40].

\section{Age Pervasiveness of Frequent Diseases}

\section{Health Tests}

Repeated, longitudinal medical laboratory workups, check-ups, to identify people who are sick without signs nor symptoms and for risk analysis of upcoming diseases are common practices in developed countries worldwide.
The major organ systems are screened with ideal labanalyses and biomarkers using the 4 major sectors of medical laboratory assays, comprising clinical chemistry, haematology, immunology, and microbiology, embraced by genome sequencing techniques that are routinely in use. Whole-genome sequencing of microbial organisms may distinguish virulent from non-virulent and antibiotic-resistant from non-resistant varieties of the same species and, thus, can be listed in personal big data banks including microbiological pathology.

The CA of patients suffering from definite nosological entities is best followed by consulting statistics on lethal diagnostics classified into age groups. A convenient classification can be made between survivors (age of onset of less than 80 years for at least one of the diseases), delayers (age of onset between the age of 80 and 100 years) and escapers (age of onset of 100 years or has not yet been diagnosed with a disease) [41]. It is well known that among them, the very old subsequently succumb to cardiovascular disease due to myocardial ageing rather than cancer $[42,43]$. Lifetime diagnoses of 10 major lethal diseases (hypertension, cardiovascular diseases, diabetes, stroke, non-skin cancer, skin cancer, osteoporosis, thyroid condition, Parkinson's disease and chronic obstructive pulmonary disease [COPD]) were listed in centenarians and the original data published at the outset of the XXI century, revealing most of the 424 centenarians (aged 97-119 years) delayed or escaped heart disease, non-skin cancer and stroke, allowing survival into the $>100$ years category [41]. The majority of these 10 lethal diseases is now on a good medical and lab-control. Because of multimorbidity accumulating at high age, these observations must be taken as preliminary. Centenarians have successfully coped with age-associated diseases. Such is the case mainly for frailty (infirmity) syndrome, sarcopenia, COPD, cancer, neurodegenerative diseases, macular degeneration, rheumatoid arthritis and osteopenia [44], which raises a speculative question: would senescence fence given diseases?

The stage for successful ageing can be explored by longitudinal follow-ups that aim to compare the health status of the very old to that of the same individual several decades prior. For example, the Georgia Centenarian Study, GCS, allowed the separation of distinct morbidity profiles, of whom cancer struck centenarians in their sixties, cardiovascular disease in their seventies and dementia in their eighties and beyond $[45,46]$. It remains speculative to derive from such observations that old age is a shelter for distinct ICD classified diseases.

The survivor, delayer, and escaper avenues may be based on different centenarian genotypes that are useful 
Fig. 2. Gross blend of frequent diseases and age groups of their predilection. The blurb positions are placed on the basis of long term international clinical observations; robust odds ratios are not available. Most of the depicted diseases occur at any age the blurb positions denote a proclivity. CVD, cardiovascular diseases; T1, type 1 diabetes mellitus; T2, type 2 diabetes mellitus; ALL, acute lymphoblastic leukaemia; AML, acute myeloblastic leukaemia; CML, chronic myelogenous leukaemia; MM, multiple myeloma; COPD, chronic obstructive pulmonary disease; MS, multiple sclerosis. The choice of the 10 selected disorders is arbitrary; the following literature references have been chosen to support the schematic [122-126].

Table 2. Age-dependent deviations of reference intervals of routine medical laboratory parameters

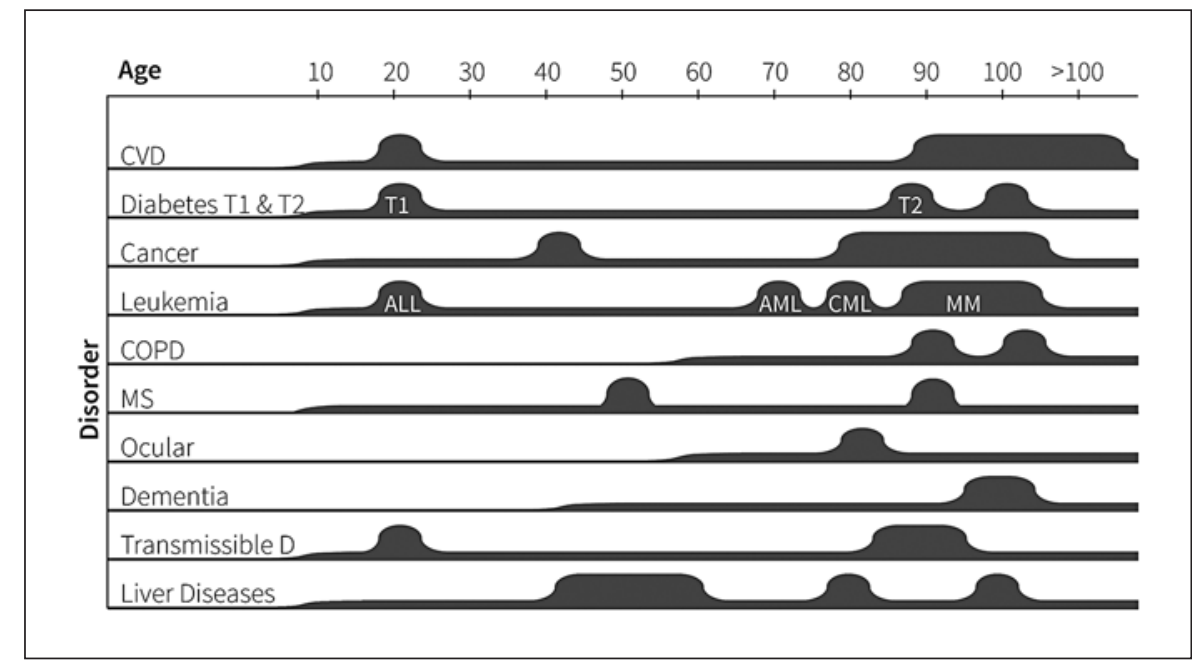

\begin{tabular}{ll}
\hline Elevated in the elderly & Decreased in the elderly \\
\hline Alkaline phosphatase & Calcium, zinc \\
Cholesterol & Creatinine kinase, eGFR* \\
Clotting factors VII and XIII & $\begin{array}{l}\text { Dehydroepiandrosterone, Testosterone } \\
\text { Oestrogen }\end{array}$ \\
D-Dimer* & Growth hormone \\
Ferritin* & IGF-1, interleukin-1* \\
Fibrinogen & Phosphor, selenium, thiamine \\
Postprandial glucose & X-tocopherol (vitamin E) \\
Parathormone & Vitamins B6 and B12, vitamin C, and vitamin D \\
IL-6* & Alanine Aminotransferase \\
Noradrenalin & \\
Parathyroid hormone & \\
Prostate specific antigen & \\
Triglycerides & \\
Uric acid & \\
HbAlc & \\
\hline
\end{tabular}

IL-6, interleukin-6; HbA1c, glycated haemoglobin. * More recent listings. to study factors that determine exceptional longevity. Physical health factors that are considered to be risk factors in midlife could actually be protective factors in very late life, for example, body weight, blood pressure and cholesterol levels (an easy and cheap lab-parameter), as well as higher body fat, as noted in some centenarian research reporting such a paradox in physically healthy very old adults particularly with regard to hypertension [47]. This observation fits well with the analysis of leading causes of death by age groups in Switzerland. The charts can be downloaded (https://www.bfs.admin.ch/bfs/de/ home/statistiken/kataloge-datenbanken/grafiken.assetdetail.262901.html).

Laboratory Assays as Biomarkers of Ageing
With extension of life expectancy, one assists to a chronological sequence of some frequent diseases shifting to the right, whereas some nosological entities do not affect the very old (Fig. 2) [41]. The laboratory medicine community began to scrutinize RIs (reference intervals) applicable for validating assays ordered for older patients. There are already short lists of analytes available for RIs that substantially deviate from work force age groups. It has been long known that sex hormones decline and a number of parameters join an ever-growing list, often immediately upon initial recognition of their importance in the improvement of diagnosis (Table 2). The SENIORLAB study initiated at our institution was able to recruit 1,467 apparently healthy elderly individuals $>60$ years of 
age; ongoing evaluations may be found at www.seniorlabor.ch [12]. Our observations are in line with previous studies of levels of laboratory analytes in different age groups and RIs, as recently summarized by Maria Edvardsson (Edvardsson [125]); the papers associated with this thesis can be located at http://urn.kb.se/resolve?urn= urn:nbn:se:liu:diva-160148. SENIORLAB is an ongoing prospective observational study, initially conceived to investigate reference intervals of routine medical laboratory in 1,467 healthy elderly study participants $\geq 60$ years of age [12], allowed to focus on participant subsets to assess organ-specific laboratory assays (Table 2).

Skin and neurological organs are at interest in biology of senescence because we have learned how to monitor age-associated changes. Next to wear and tear, skin senescence shows itself in the subcutis with loosening of collagen fibres and reduced proliferation of cells in the basal cell layer [48]. An age-dependent increase in the expression of the senescence marker $\beta$-galactosidase in dermal fibroblasts and epidermal keratinocytes is notable [49]. So far, no link between skin senescence and immunosenescence has been found to portend that local, organ-specific senescence prevails over systemic retarding of the course of senescence by recirculating immune cells or stem cells. Skin cells can be rejuvenated, for example, by transforming then into hIPSC [50].

Hair follicle stem cells that are not contributing to epidermal homeostasis will exit their niche and participate in repopulating the epidermis: the Sartorius \& Science Prize Winner, Dr. Yaron Fuchs, has recently summarized such adjuvant repair features in his prize essay [39]. This work allows one to understand the phenomenon of apoptosis as repair assistance: stress and injury lead to mitogenic and morphogenic features to stimulate growth and repair in the apoptotic cell surroundings; apoptosis is not merely a deadline of senescence but would bear potential for rejuvenation. Cellular senescence is a focus of neural progenitor cells (NPCs) because its exploration might directly allow a better understanding of diseases such as primary progressive multiple sclerosis (PPMS): senescent cells are found in remyelinated white matter lesion autopsy tissue, and cellular senescence markers are found in abundance. This can be treated with rapamycin to restore primary progressive multiple sclerosis neural progenitor cell-mediated support for oligodendrocyte maturation [51]. Thus, although MS was not perceived as a disease of old age previously, the growing segment of individuals aged $\geq 80$ years allows us to perceive a shift of multiple sclerosis into the elderly population [52] (Fig. 2).
Senescent cells accumulate with age in a variety of human and mouse tissues where they express a complex "senescence-associated secretory phenotype" (see below). The SASP includes many pro-inflammatory cytokines, chemokines, growth factors and proteases that have the potential to cause or exacerbate age-related pathology, both degenerative and hyperplastic. While cellular senescence in peripheral tissues has recently been linked to a number of age-related pathologies, its involvement in brain ageing is just beginning to be explored. Age-related neurodegenerative diseases are accompanied by an increase in SASP-expressing senescent cells of non-neuronal origin in the brain corresponding to neurodegeneration. Senescent cells in the brain constitute novel therapeutic targets for treating age-related neuropathologies. In the adult brain, cognitive and regenerative impairments have been subjected to experiments using heterochronic parabiosis, in which the circulatory systems of young and old animals are joined [53]. $\beta 2$-microglobulin has been observed to negatively regulate cognitive function in the adult hippocampus in an age-dependent manner, and an imbalance of the 2 endogenous metals copper and zinc has been suggested to propel cognitive decline [54].

More than any other organ, brain senescence exhibits extensive variation. Different brachistochrone curves in some people with early mental decline may be followed by steady holding periods. Long-term steady alertness may be forfeited only in late life. In both instances, similar mental cognitive test scores may result. Many believe that discrepancies between brain-predicted age and chronological age would reflect predementia for a given age. The University of Edinburgh Centre for Cognitive Ageing and Cognitive Epidemiology has comprehensively reviewed these aspects and placed them in perspective to a series of biomarkers, including DNAm, telomere length, sphingolipids, and protein glycosylation extent [55].

Age-related neurodegenerative diseases have been found to be accompanied by an increase in SASP-expressing senescent cells (Table 1) of non-neuronal origin in the brain, a finding that correlates with neurodegeneration. It has been reported that senescence $=\mathrm{e}$ of neurological tissue proceeds on its own and is independent from other organs of the same individual [56].

\section{Metabolic Profile}

Somatic cells, down to their senescent state, rely on metabolic energy expenditure; the metabolome impacts the epigenome with the potential to control cell fate [57]. Glycation of proteins occurs in physiological systems to a 
low extent - typically 1-10 mol percent. Glycation by glucose forms N-terminal and lysyl side chain N-1 deoxyfructosyl residues or fructosamines, which are exploited clinically for the assessment of glycaemic control by measurement of glycated haemoglobin (HbAlc). A further major type of protein glycation is by methylglyoxal (MG) - a reactive dicarbonyl metabolite formed from intermediates. Methylglyoxal modifies proteins, misfolding and inactivating them [58]. Non-enzymatic glycation of proteins through the Maillard reaction is a physiological process, the extent of which impacts protein interactions with specific receptor (auto-) antibodies, protein halflives, senescence, and protein folding. Thus, prediabetes, a pre-disease status, may be quantitatively estimated by measuring glycated $\mathrm{HbAlc}$ and fructosamine [13]. Biomarkers that might best gauge DALY are now being increasingly explored (http://mortalitypredictors.org) [59].

\section{Ageing, Hallmarks, and Biomarkers}

Ageing, the time dependent deterioration of those physiological processes of the organism that support its survival and fertility, is complemented by senescence, a cue that is better characterized at a cellular level by an irreversible form of cell-cycle delay [22,60-63]. Definitions of ageing are subject to discussion but also retain coherency with the view of ageing as due to the plateauing of natural selection forces [64].

There is a long tradition of evolving views and theories of ageing. Endeavours abound to conceptually clarify and theoretically contrast with each other the various theories such as evolutionary, non-evolutionary, programmed and non-programmed [65-68]. Harmonization efforts are underway, for example, using emerging new concepts such as the "deleteriome" [69], unified mechanistic views [70] and the "somatic restriction theory of ageing," with the latter linking the "antagonist pleiotropy" theory to modern observations of epigenetics regulation in ageing, potentially leading to clinical applications in regenerative medicine [71].

The definition of ageing and its theories goes hand-inhand with a more sophisticated definition of "state of health." Together with the concept of "absence of disease," which is probably undergoing disuse and likely a too idealistic view of the state of "complete physical, mental and social well-being" (WHO), the more recent concepts of homeostasis, allostasis and "allostatic load" have emerged; they might better capture the organ and system dynamic adaptations to stressors and the environment by

Laboratory Assays as Biomarkers of Ageing examining the capabilities of the organ or system when reacting to perturbation [72]. Receptor-binding domains sensitive to the spike protein (S) of SARS-CoV-2 are abound on respiratory lining cells and with the concept of allostasis, that is, different types of organ resilience exceeds the lung - as exemplified by the liver with its "capability" to regenerate [73]. There have been interesting attempts to unify in a conceptual frame both ageing and health, which might potentially lead to precision prevention interventions [24]. A wide spectrum of clinical manifestations, similar for systemic autoimmune disease, show off in SARS-CoV-2; this makes us believe that stress on the immune system is a prime pathogenic event in this disease [74].

Despite the complexity of senescence, a small number of substantial molecular and cellular changes determine the "hallmarks of ageing," which bridge links to various diseases and can be potentially amenable to interventions [75-78]. These interventions can possibly be classified into broad categories such as systemic blood components and metabolic manipulations, potentially mimicking calorie or dietary restrictions, senolytics (drugs removing senescence cells) and cellular reprogramming [79].

The necessity for a metrics of senescence and ageing with biomarkers goes along with the disentanglement of chronological and biological points in time. CA is disentangled from $\mathrm{BA}$, a measure of the degree of age-associated decline that an individual undergoes [80-83]. Within this scope, routine medical laboratory assays can be leveraged as part of modern high-throughput technologies for genomics, transcriptomics, proteomics, microbiomics and metabolomics, hence contributing to the integrative analysis of biological events such as senescence [84].

Ideal biomarkers should be able to predict individual age-specific mortality and age-associated pathology in addition to and better than chronological age alone, and they should serve as metrics and risk factors $[83,85]$. Such requirements also reflect challenges in choosing the right biomarkers. As a biomarker of BA is expected to be correlated to $\mathrm{CA}$, one is tempted to use the highest correlation as a selection criterion, but then one might also question the necessity of using a biomarker other than typical for CA at all $[86,87]$ This conflict has been referred to as the "biomarkers paradox". Applications other than geriatrics are forensic or racial in determining an unknown CA or to assess the state of health of an individual/a patient. Moreover, as the biological heterogeneity present in old population increases relative to periods of lower CA, the "positive predictive value" of a specific biomarker is expected to weaken [88]. 


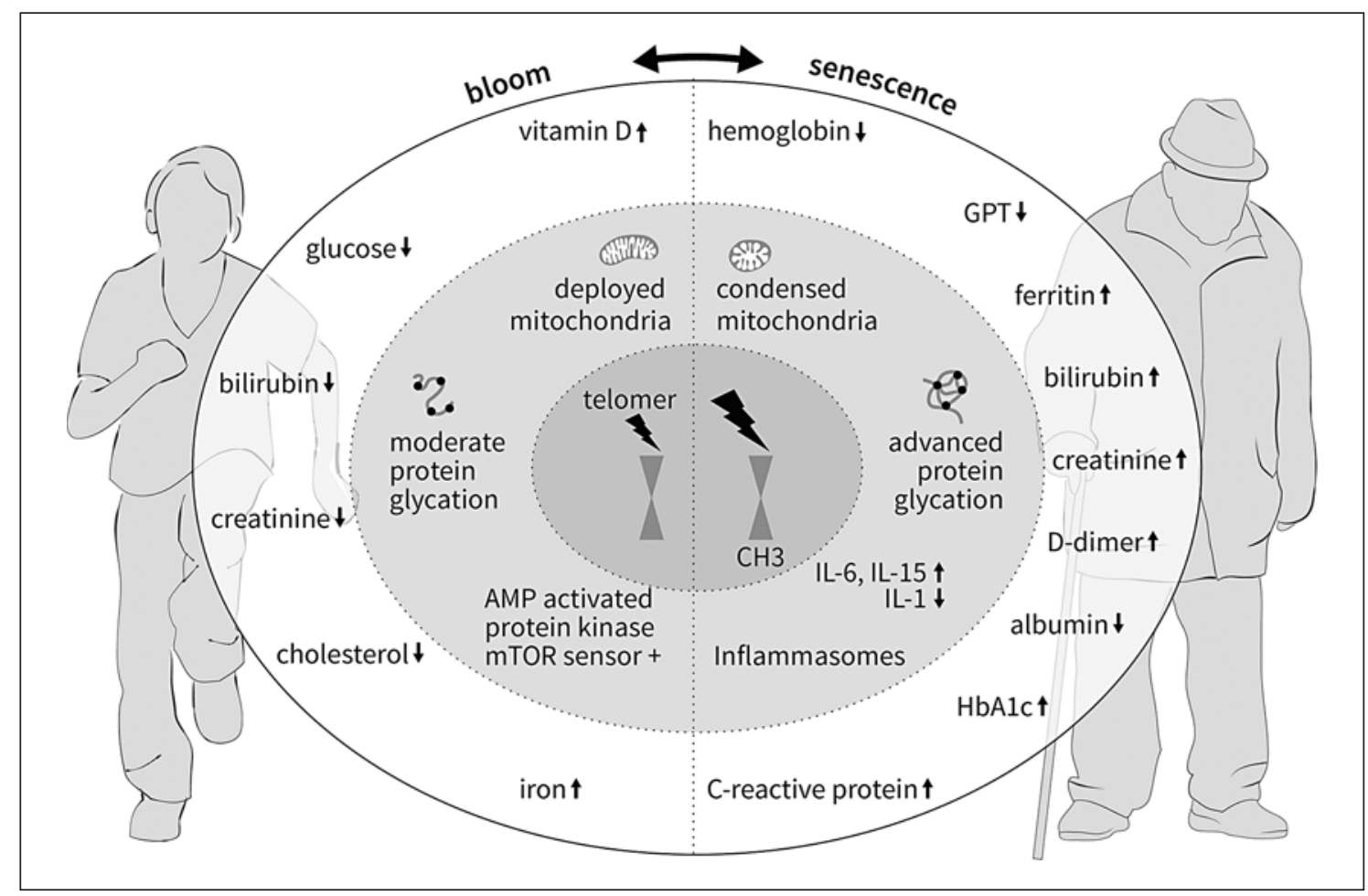

Fig. 3. Alignment of elementary medical laboratory tests over biologic features of senescence. Central edging: cell nucleus showing telomere shortening and DNAm [127, 128]. Grey nuclear surrounding: acknowledged markers of transition from bloom to senescence. White outer surrounding: arbitrarily selected routine medical laboratory assays with putative biomarker status related to underlying biochemistry.

Here, we attempt to outline a flavour of the variety of approaches towards a metric of ageing that is far from being complete. Described as a quest [89], the importance of the search for a useful metric of BA is witnessed by the large number of PubMed articles, estimated at approximately 1,500 over the past 2 decades [87], and focused efforts such as the European Commission funded MARK-AGE project [90] aimed at identifying and combining a set of biomarkers of various nature measuring BA.

Several "hallmarks" and "pillars" of ageing $[76,78,91]$ have been proposed and are frequently quoted in the literature, including genomic instability, telomere attrition, epigenetic alterations, loss of proteostasis, deregulated nutrient sensing, mitochondrial dysfunction, cellular senescence (cell cycle arrest, [22]), stem cell exhaustion and altered intercellular communication. They can be grouped into primary, antagonistic and integrative hallmarks, all of which impact both anabolic and catabolic metabolism and control longevity $[76,78]$. For each hallmark of ageing specific biomarkers in humans have been proposed $[89,92-94]$. The biological causes or hallmarks of health include features spatial compartmentalization, maintenance of homeostasis over time, and adequate responses to stress; disruption of any of these interlocked features is broadly pathogenic [76].

The hallmarks of ageing can also provide a guide to the development of proxies of BA, which have great potential for translational research and in the clinical setting. Much work remains to be done, but several approaches might be implementable already. DNAm, has recently emerged as the base of the so-called epigenetic clock. DNAm is interpreted as a precise marker of BA, showing a strong association with multi-morbidity, health span, lifespan, frailty [95] and mortality [45, 96-99].

The human immune system and its senescence (immunosenescence) are of paramount importance in ageing. In a relevant study, multi-omics technologies were used in longitudinally tracking over 9 years in 135 healthy adults. A high inter-individual heterogeneity was observed, showing that each individual ages differently. The data formed a higher dimensional trajectory marker (IMM-AGE) describing individual immune system status better than CA [100]. 
A particular note is required for "inflammaging" (chronic, sterile, low-grade inflammation), classified under the hallmark of altered cellular communication [76, 78]. Inflammation as a driver of ageing, or "inflammaging" $[44,101]$ seems to be directly or indirectly caused by many of the hallmarks of ageing, potentially capturing the global burden of the ageing process [89, 102]. Efforts have been made towards to acquire well known and new biomarkers to create indices of BA. As an example, items such as symptoms, signs, key routine labs, disease classification and disabilities were merged into a frailty index (FI) interpreted as a proxy of global ageing. The FI, defined as "a ratio of the number of health deficits that individuals accumulated to the total number of deficits available in the database or the study" was introduced in 2001 by Mitnitski and Rockwood [87] and later developed by the authors. Along the lines of our tentative functional definition of ageing, several panels have been defined by experts and include biomarkers of physiological, endocrine, physical, cognitive, and immune functions [103].

One approach that seems particularly relevant to the authors is using the widely available, relatively inexpensive, standardized and clinically validated routine laboratory analytes designed as biomarkers that are very similar to those used in the authors' SENIORLAB work [12]. Additional information may stem from ratio calculation of related analytes $[13,104]$. These biomarkers, available in larger population cohorts, can be composited and analysed to extract signatures of BA, which can be compared with CA. In Figure 3, we attempt to align a partial list of these biomarkers to detailed characteristics of cellular senescence.

Using data from the Long Life Family Study (LLFS) cohort, 19 routine laboratory biomarkers (high-sensitivity C-reactive protein, interleukin 6, N-terminal B-type natriuretic peptide, absolute monocyte count, white blood cell counts, red blood cell distribution width, transferrin receptor, mean corpuscular volume, haemoglobin [Hgb], glycated haemoglobin [HbAlc], soluble receptor for advanced glycation end product [sRAGE], adiponectin [Adip], insulin-like growth factor [IGF1], total cholesterol [T.Chol], sex hormone-binding globulin [SHBG], dehydroepiandrosterone sulfate [DHEA], albumin [Album], creatinine, cystatin) were able to identify signatures associated with important ageing-related physiological functions and to some extent predict changes in physical and cognitive function, survival, and risk of cancer, CVD and T2DM diseases [105]. When similar biomarkers data from the NHANES III cohort were used,
Morgan Levine used the Klemera \& Doubal regression algorithm [106] to predict mortality, which may be better than other algorithms and more informative. The algorithms can also be improved by integrating biomarkers of different nature (e.g., anthropometric) in a single measure [107]. Levine then established the concept of "Phenotypic Age" and validated the association to all-cause and single cause-specific mortality, nosological entity counts and physical functioning, all together predicting both morbidity and mortality [97, 108]. Phenotypic age was calculated using CA and 9 biomarkers that are readily available in standard routine labs (albumin, creatinine, glucose, [log] CRP, lymphocyte percent, mean corpuscular volume, red blood cell distribution width, alkaline phosphatase and white blood cell count) using a Cox proportional hazard elastic net model for mortality and "parametrization of 2 Gompertz proportional hazard models - one fit using all 10 selected variables, and the other fit using only chronological age" [108]. Moreover, using data from the CHIANTI cohort and regressing the phenotypic age on blood DNAm data, Levine developed a new epigenetic biomarker of phenotypic age called "DNAm PhenoAge" [97]. The phenotypic age was also recently included in a longitudinal examination of ageing at individual level showing a variety of ageing patterns or "ageotypes" [109].

In a work based on 1,013 participants in the Canadian Study of Health and Aging, a head-to-head comparison of 2 measures of BA with 3 frailty indices (FI) was proposed, one of which was constructed from standard laboratory blood tests and blood pressure. One interesting aspect of the study was the non-inclusion of CA in the formulas determining the difference between BA and CA [86]. Relationships between ageing, frailty and mortality were further explored using a computational model based on nodes of a complex network [110].

In a recent DNAm study using a slightly different analytical strategy, a new high-performance clock (called GrimAge) [111] was designed by combining CA, sex, and DNAm-based surrogate biomarkers for a distinct selection of blood plasma proteins (out of an initial 88 measured by immunoassay) and smoking pack years. An ageadjusted version of DNAm GrimAge was found to be associated with a host of age-related conditions [112], lifestyle factors, and clinical biomarkers. It was shown to be particularly predictive of time to death, time to coronary heart disease and time to cancer $[111,113]$.

As the number of biomarkers and different measurement technologies is expected to increase in the coming years, the use of AI-driven techniques is also expected to 
Fig. 4. On the radar of senescence. Six major influencers of senescence weighed in a spider web putatively customized to individual profiles. Escaper, delayer and survivor profiles may differ as shown Telomere shortening prevails in each of the putative elderly subjects, but in B, lifestyle can override senescence biology. In $\mathrm{C}$, genetic predisposition might be unequivocal in setting the pace of senescence.

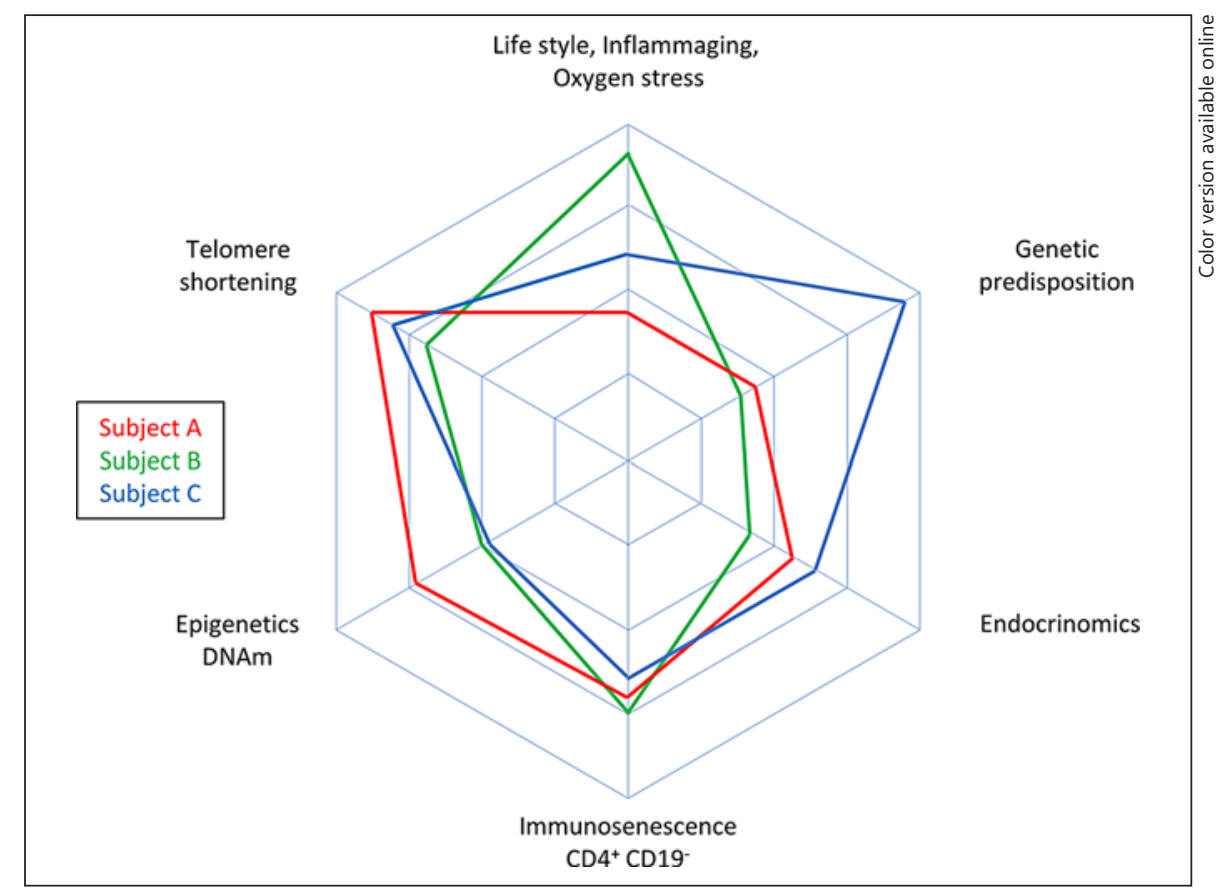

The challenges introduced by reliable BA metrics addressing most, if not all, aspects of the human ageing process are motivating. The rewards of prolonging human health span, that is, reducing DALY, by slowing the ageing process will likely provide substantial economic returns for humanity [116].

\section{Alignment of Deep Senescence Markers with a Routine Laboratory Assay}

The effect of ageing on the homeostasis of the biological systems allows the recognition of some trends [100, 110].

Support from life clocks based on epigenetic characteristic might help to improve metrics of senescence. Experimental laboratory studies have opened new doors, but those based on elementary routine medical laboratory assays in humans of different ages lag behind.

With the prolonged life expectancy running across the 3rd decade of the XXI century, we do need large-scale accessible and cost-efficient medical laboratory assays to pursue description of health recalled by the elderly [117, 118]. An arbitrary selection of these routine assays (outer circle) associated with deep characteristics of cellular senescence and ageing (inner circle) is tentatively presented in Figure 3. 
Major influencers of senescence weighed in a spider web putatively customized to individual profiles are shown in Figure 4. Escaper, delayer and survivor [41] profiles may differ, as represented. Individual heterogeneity increases with age, and major influencers of senescence are depicted with their relative weights. At the population level, genetic predispositions might be more impactful in the late stages of life beyond age 70 years, and for the very old beyond age 90 years, specific longevity genes might emerge [119].

As described, several tools are available to allow public testing and scrutinize real-life performance, such as AI and the phenotypic age for non-invasively tracking of biomarkers of biological ageing. Moreover, age-associated reference intervals for distinct decennials of life manifest in clusters, as recently proposed by the Centre for Healthy Aging in Copenhagen, Denmark [120].

\section{Conclusion}

In this paper, we attempted to better link our current knowledge of human ageing, cellular and organ senescence and the appearance of diseases with the widely available and relatively inexpensive routine laboratory assays extracted from many studies including our SENIORLABOR. New parameters of interest, particularly with COVID-19 (C3a, SC5b-9, AB0 type), might arise [9]. The current biological insights into human ageing/senescence and the use of advanced computational tools and multi-omics allow us to set chronological and BAs apart, the latter being viewed as a possible quantitative way to assess the health heterogeneity of individuals and to track their health status.
We hope that these and similar approaches, in particular using routine medical laboratory assays, will continue to be improved and possibly used in the clinical setting among disease-free, healthy adults of different CAs. Additional longitudinal and comparative studies will improve our understanding of human ageing trajectories for a better translation of research in the clinic and disease prevention (this paper is an extension of a formerly published report [121]).

\section{Conflict of Interest Statement}

The co-authors have no conflicts of interest to declare. The SENIORLAB study is a registered medical laboratory survey of healthy elderly (International Standard Registered Clinical/Social Study No. 53778569).

The study benefits from in-house funding.

\section{Author Contributions}

L.R. and M.R. have designed the SENIORLAB study, performed the laboratory analyses and proposed the putative biomarker properties of routine laboratory assays. T.L., P.D.C., and U.N. have collected the SENIORLAB data for projection onto the current literature on senescence; P.D.C.: InnoBoost SA, Lausanne, Switzerland payd travel expenses during the initial phase of the manuscript elaboration. U.N. helped to write the manuscript. The figure materials were designed essentially by U.N. using Microsoft PowerPoint software and completed by Anja Giger, medical illustrator, University Hospital Inselspital Bern, Switzerland; American Journal Experts provided linguistic help.

\section{References}

1 Bajaj V, Gadi N, Spihlman AP, Wu SC, Choi $\mathrm{CH}$, Moulton VR. Aging, immunity, and COVID-19: how age influences the host immune reponse to coronavirus infections? Front Physiol. 2021 Jan 12;11: 571416.

2 Java A, Apicelli AJ, Liszewski MK, Coler-Reilly A, Atkinson JP, Kim AH, et al. The complement system in COVID-19: friend and foe? JCI Insight. 2020;5(15):e140711.

3 Blagosklonny MV. Geroconversion: irreversible step to cellular senescence. Cell Cycle. 2014;13(23):3628-35.

4 Blagosklonny MV. Disease or not, aging is easily treatable. Aging. 2018;10(11):3067-78.

5 Chang AY, Skirbekk VF, Tyrovolas S, Kassebaum NJ, Dieleman JL. Measuring population ageing: an analysis of the Global Burden of Disease Study 2017. Lancet Public Health. 2019;4(3):e159-67.

6 Mammalian Methylation Consortium; Lu AT, Fei Z, Haghani A, Robeck TR, Zoller JA, et al. Universal DNA methylation age across mammalian tîssues. bioRxiv. 2021 Jan 19.

7 Santesmasses D, Castro JP, Zenin AA, Shindyapina AV, Gerashchenko MV, Zhang B, et al. COVID-19 is an emergent disease of aging. Aging Cell. 2020;19(10):e13230.

8 Shi J, Zhang B, Choi JY, Gao YT, Li H, Lu W, et al. Age at menarche and age at natural menopause in East Asian women: a genomewide association study. Age. 2016;38(5-6): $513-23$.

9 Valenti L, Griffini S, Lamorte G, Grovetti E, Uceda Renteria SC, Malvestiti F, et al. Chromosome 3 cluster rs11385942 variant links complement activation with severe COVID-19. J Autoimmunity. 2021;117:102595.

10 Cugno M, Meroni PL, Gualtierotti R, Griffini $\mathrm{S}$, Grovetti E, Torri A, et al. Complement activation in patients with COVID-19: a novel therapeutic target. J Allergy Clin Immunol. 2020;146(1):215-7.

11 Montesanto A, Geracitano S, Garasto S, Fusco S, Lattanzio F, Passarino G, et al. The impact of the emerging genomics data on the management of agerelated phenotypes in the context of cellular senescence. Curr Drug Targets. 2016;17(4):428-38.

12 Risch M, Nydegger U, Risch L. SENIORLAB a prospective observational study investigating laboratory parameters and their reference intervals in the elderly. Medicine. 2017;96(1): e5726. 
13 Medina Escobar P, Sakem B, Risch L, Risch M, Grebhardt C, Nydegger UE, et al. Glycaemic patterns in healthy elderly individuals and in those with impaired glucose metabolism - exploring the relationship with nonglycaemic variables. Swiss Med Wkly. 2019;149:w20163.

14 Lozano R, Naghavi M, Foreman K, Lim S, Shibuya K, Aboyans V, et al. Global and regional mortality from 235 causes of death for 20 age groups in 1990 and 2010: a systematic analysis for the Global Burden of Disease Study 2010. Lancet. 2012;380(9859):2095-128.

15 Calimport SRG, Bentley BL. Aging classified as a cause of disease in ICD-11. Rejuvenation Res. 2019;22(4):281.

16 Genedat M, Tarancon-Diez K, de Pablo-Bernal R, Calderón A, Muñoz-Fernández MÁ, Leal M. Coronavirus disease (COVID-19): a perspective from immunosenescence. Aging Dis. 2021;12(1):3-6.

17 Zhavoronkov A, Mamoshina P, Vanhaelen Q, Scheibye-Knudsen M, Moskalev A, Aliper A. Artificial intelligence for aging and longevity research: recent advances and perspectives. Ageing Res Rev. 2019;49:49-66.

18 Zhavoronkov A, Bhullar B. Classifying aging as a disease in the context of ICD-11. Front Genet. 2015;6:326.

19 The Lancet Diabetes Endocrinology. Opening the door to treating ageing as a disease. Lancet Diabetes Endocrinol. 2018;6:587.

20 Fulop T, Larbi A, Khalil A, Cohen AA, Witkowski JM. Are we ill because we age? Front Physiol. 2019;10:1508.

21 Magalhaes JP, Passos JF. Stress, cell senescence and organismal ageing. Mech Ageing Dev. 2018;170:2-9.

22 Calcinotto A, Kohli J, Zagato E, Pellegrini L, Demaria M, Alimonti A. Cellular senescence: aging, cancer, and injury. Physiol Rev. 2019; 99(2):1047-78

23 Gems D. The aging-disease false dichotomy: understanding senescence as pathology. Front Genet. 2015;6:212.

24 Fuellen G, Jansen L, Cohen AA, Luyten W, Gogol M, Simm A, et al. Health and aging: unifying concepts, scores, biomarkers and pathways. Aging Dis. 2019;10(4):883-900.

25 Rautschert E. Survivorship curves. Nat Education Knowledge. 2010;3(10):18.

26 Weon BM, Je JH. Trends in scale and shape of survival curves. Sci Rep. 2012;2:504.

27 Meer VM, Podolskiy DI, Tyshkovskiy A, Gladyshev VN. A whole lifespan mouse milti-tissue DNA methylation clock. Elife. 2018;7: e40675.

28 Childs BG, Durik M, Baker DJ, van Deursen JM. Cellular senescence in aging and age-related disease: from mechanisms to therapy. Nat Med. 2015;21(12):1424-35.

29 Laberge RM, Sun Y, Orjalo AV, Patil CK, Freund A, Zhou L, et al. MTOR regulates the pro-tumorigenic senescence-associated secretory phenotype by promoting IL1A translation. Nat Cell Biol. 2015;17(8):1049-61.

30 Richardson NE, Konon EN, Schuster HS, Mitchell AT, Boyle C, Rodgers AC, et al. Life- long restriction of dietary branched-chain amino acids has sex-specific benefits for frailty and lifespan in mice. Nat Aging. 2021;1(1): 73-86.

31 Bischof E, Siow RC, Zhavoronkov A, Kaeberlein $\mathrm{M}$. The potential of rapalogs to enhance resilience against SARS-CoV-2 infection and reduce the severity of COVID-19. Lancet Healthy Longev. 2021;2(2):E105-11.

32 Mannick JB, Del Giudice G, Lattanzi M, Valiante NM, Praestgaard J, Huang B, et al. mTOR inhibition improves immune function in the elderly. Sci Transl Med. 2014;6(268): 268 ra179.

33 Lau L, Porciuncula A, Yu A, Iwakura Y, David G. Uncoupling the senescence-associated secretory phenotype from cell cycle exit via interleukin-1 inactivation unveils its protumorigenic role. Mol Cell Biol. 2019;39(12):e00586-18.

34 Hall BM, Balan V, Gleiberman AS, Strom E, Krasnov P, Virtuoso LP, et al. Aging of mice is associated with p16(Ink4a)- and beta-galactosidase-positive macrophage accumulation that can be induced in young mice by senescent cells. Aging. 2016;8(7):1294-315.

35 Storer M, Mas A, Robert-Moreno A, Pecoraro M, Ortells MC, Di Giacomo V, et al. Senescence is a developmental mechanism that contributes to embryonic growth and patterning. Cell. 2013;155(5):1119-30.

36 Debacq-Chainiaux F, Erusalimsky JD, Campisi J, Toussaint O. Protocols to detect senescenceassociated beta-galactosidase (SA-betagal) activity, a biomarker of senescent cells in culture and in vivo. Nat Protoc. 2009;4(12):1798-806.

37 Lawless C, Wang C, Jurk D, Merz A, Zglinicki T, Passos JF. Quantitative assessment of markers for cell senescence. Exp Gerontol. 2010;45(10):772-8.

38 Pollock SB, Hu A, Mou Y, Martinko AJ, Julien $\mathrm{O}$, Hornsby M, et al. Highly multiplexed and quantitative cell-surface protein profiling using genetically barcoded antibodies. Proc Natl Acad Sci U S A. 2018;115(11):2836-41.

39 Fuchs Y. The therapeutic promise of apoptosis. Science. 2019;363(6431):1050-1.

40 Armenian SH, Gibson CJ, Rockne RC, Ness KK. Premature aging in young cancer survivors. J Natl Cancer Inst. 2019;111(3):226-32.

41 Evert J, Lawler E, Bogan H, Perls T. Morbidity profiles of centenarians: survivors, delayers, and escapers. J Gerontol A Biol Sci Med Sci. 2003;58(3):232-7.

42 Pavlidis N, Stanta G, Audisio RA. Cancer prevalence and mortality in centenarians: a systematic review. Crit Rev Oncol Hematol. 2012;83(1):145-52.

43 Ramos GC, van den Berg A, Nunes-Silva V, Weirather J, Peters L, Burkard M, et al. Myocardial aging as a T-cell-mediated phenomenon. Proc Natl Acad Sci U S A. 2017;114(12): E2420-9.

44 Franceschi C, Garagnani P, Morsiani C, Conte M, Santoro A, Grignolio A, et al. The continuum of aging and age-related Diseases: common mechanisms but different rates. Front Med. 2018;5:61.
45 Arnold B, Mitnitski A. Epigenetic biomarkers for biological age. Translational Epitgenetics. 2018;4:153-70.

46 Arnold J, Dai J, Nahapetyan L, Arte A, Johnson MA, Hausman D, et al. Predicting successful aging in a population-based sample of georgia centenarians. Curr Gerontol Geriatr Res. 2010;2010:989315.

47 Martin P, Gondo Y, Arai Y, Ishioka Y, Johnson MA, Miller LS, et al. Cardiovascular health and cognitive functioning among centenarians: a comparison between the Tokyo and Georgia centenarian studies. Int Psychogeriatr. 2019;31(4):455-65.

48 Zhang S, Duan E. Fighting against skin aging: the way from bench to bedside. Cell Transplant. 2018;27(5):729-38.

49 Dimri GP, Lee X, Basile G, Acosta M, Scott G, Roskelley C, et al. A biomarker that identifies senescent human cells in culture and in aging skin in vivo. Proc Natl Acad Sci U S A. 1995; 92(20):9363-7.

50 Waaijer MEC, Goldeck D, Gunn DA, van Heemst D, Westendorp RGJ, Pawelec G, et al. Are skin senescence and immunosenescence linked within individuals? Aging Cell. 2019; 18(4):e12956

51 Nicaise AM, Wagstaff LJ, Willis CM, Paisie C, Chandok H, Robson P, et al. Cellular senescence in progenitor cells contributes to diminished remyelination potential in progressive multiple sclerosis. Proc Natl Acad Sci U S A. 2019;116(18):9030-9.

52 Sanai SA, Saini V, Benedict RH, Zivadinov R, Teter BE, Ramanathan M, et al. Aging and multiple sclerosis. Mult Scler. 2016;22(6): 717-25.

53 Smith LK, He Y, Park JS, Bieri G, Snethlage $\mathrm{CE}$, Lin $\mathrm{K}$, et al. $\beta 2$-microglobulin is a systemic pro-aging factor that impairs cognitive function and neurogenesis. Nat Med. 2015; 21(8):932-7.

54 Sensi SL, Granzotto A, Siotto M, Squitti R. Copper and zinc dysregulation in Alzheimer's disease. Trends Pharmacol Sci. 2018;39(12): 1049-63.

55 Cole JH, Marioni RE, Harris SE, Deary IJ. Brain age and other bodily "ages": implications for neuropsychiatry. Mol Psychiatry. 2019;24(2):266-81.

56 Chinta SJ, Woods G, Rane A, Demaria M, Campisi J, Andersen JK. Cellular senescence and the aging brain. Exp Gerontol. 2015;68: 3-7.

57 Gatie MI, Kelly GM. Metabolic profile and differentiation potential of extraembryonic endoderm-like cells. Cell Death Discov. 2018; $4: 42$.

58 Rabbani N, Thornalley PJ. Advanced glycation end products in the pathogenesis of chronic kidney disease. Kidney Int. 2018; 93(4):803-13.

59 Peto MV, De la Guardia C, Winslow K, Ho A, Fortney K, Morgen E. MortalityPredictors. org: a manually-curated database of published biomarkers of human all-cause mortality. Aging. 2017;9(8):1916-25. 
60 Strehler BL. Understanding aging. Methods Mol Med. 2000;38:1-19.

61 Dodig S, Čepelak I, Pavić I. Hallmarks of senescence and aging. Biochem Med. 2019; 29(3):030501.

62 Noren Hooten N, Evans MK. Techniques to induce and quantify cellular senescence. J Vis Exp. 2017;(123):55533.

63 Zeng Y, Nie C, Min J, Chen H, Liu X, Ye R, et al. Sex differences in genetic associations with longevity. JAMA Netw Open. 2018;1(4): e181670.

64 Flatt T. A new definition of aging? Front Genet. 2012;3:148.

65 Libertini G. Sex and aging: a comparison between two phenoptotic phenomena. Biochemistry. 2017;82(12):1435-55.

66 Ljubuncic P, Reznick AZ. The evolutionary theories of aging revisited: a mini-review. Gerontology. 2009;55(2):205-16.

67 Libertini G, Rengo G, Ferrara N. Aging and aging teories. J Gerontol Geriatrics. 2017:659.

68 da Costa JP, Vitorino R, Silva GM, Vogel C, Duarte AC, Rocha-Santos T. A synopsis on aging-theories, mechanisms and future prospects. Ageing Res Rev. 2016;29:90-112.

69 Gladyshev VN. Aging: progressive decline in fitness due to the rising deleteriome adjusted by genetic, environmental, and stochastic processes. Aging Cell. 2016;15(4):594-602.

70 Barja G. Towards a unified mechanistic theory of aging. Exp Gerontol. 2019;124:110627.

71 West MD, Sternberg H, Labat I, Janus J, Chapman KB, Malik NN, et al. Toward a unified theory of aging and regeneration. Regen Med. 2019;14(9):867-86.

72 Sholl J, Rattan SIS. Biomarkers of heatlh and healthy ageing from the outside-in. In: Moskalev A, editors. Biomarkers of human aging, heatlhy ageing and longevity. Springer Cham; 2019.

73 Morsiani C, Bacalini MG, Santoro A, Garagnani P, Collura S, D'Errico A, et al. The peculiar aging of human liver: a geroscience perspective within transplant context. Ageing Res Rev. 2019;51:24-34.

74 Woodruff MC, Ramonell RP, Nguyen DC, Cashman KS, Saini AS, Haddad NS, et al. Extrafollicular B cell responses correlate with neutralizing antibodies and morbidity in COVID-19. Nat Immunol. 2020;21(12): 1506-16.

75 De Grey AD, Ames BN, Andersen JK, Bartke A, Campisi J, Heward CB, et al. Time to talk SENS: critiquing the immutability of human aging. Ann N Y Acad Sci. 2002;959:452-5.

76 Lopez-Otin C, Blasco MA, Partridge L, Serrano M, Kroemer G. The hallmarks of aging. Cell. 2013;153(6):1194-217.

77 Kennedy BK, Berger SL, Brunet A, Campisi J, Cuervo AM, Epel ES, et al. Geroscience: linking aging to chronic disease. Cell. 2014; 159(4):709-13

78 Lopez-Otin C, Galluzzi L, Freije JMP, Madeo F, Kroemer G. Metabolic control of longevity. Cell. 2016;166(4):802-21.
79 Mahmoudi S, Xu L, Brunet A. Turning back time with emerging rejuvenation strategies. Nat Cell Biol. 2019;21(1):32-43.

80 George L, Korstanje R. Longevity as a complex genetic trait. In: Kaeberlein M, Martin GM, editor. Handbook of the biology of aging. 8th ed. Academic Press; 2016.

81 Kaeberlein M. The biology of aging: citizen scientists and their pets as a bridge between research on model organisms and human subjects. Vet Pathol. 2016;53(2):291-8.

82 Kirkwood TB. Alex Comfort and the measure of aging. Exp Gerontol. 1998 Jan-Mar;33(12):135-40.

83 Butler RN, Sprott R, Warner H, Bland J, Feuers R, Forster M, et al. Biomarkers of aging: from primitive organisms to humans. J Gerontol A Biol Sci Med Sci. 2004;59(6): B560-7.

84 Khan S, Ince-Dunn G, Suomalainen A, Elo LL. Integrative omics approaches provide biological and clinical insights: examples from mitochondrial diseases. J Clin Invest. 2020; 130(1):20-8.

85 Gutzwiller JP, Richterich JP, Stanga Z, Nydegger UE, Risch L, Risch M. Osteoporosis, diabetes, and hypertension are major risk factors for mortality in older adults: an intermediate report on a prospective survey of 1467 community-dwelling elderly healthy pensioners in Switzerland. BMC Geriatr. 2018; 18(1):115

86 Mitnitski A, Howlett SE, Rockwood K. Heterogeneity of human aging and its assessment. J Gerontol A Biol Sci Med Sci. 2017; 72(7):877-84

87 Mitnitski A, Rockwood K. The problem of integrating of biological and clinical markers of aging. In: Moskalev A, editors. Biomarkers of human aging, healthy ageing and longevity. Springer, Cham; 2019.

88 Jylhava J, Pedersen NL, Hagg S. Biological age predictors. EBioMedicine. 2017;21:29-36.

89 Ferrucci L, Gonzalez-Freire M, Fabbri E, Simonsick E, Tanaka T, Moore Z, et al. Measuring biological aging in humans: a quest. Aging Cell. 2020;19(2):e13080.

90 Bürkle A, Morenon-Villanueva M, Bernhard J. MARK-AGE biomarkers of ageing. Mech Ageing Dev. 2015;151:2-12.

91 Sierra F. The emergence of geroscience as an interdisciplinary approach to the enhancement of health span and life span. Cold Spring Harb Perspect Med. 2016;6(4):a025163.

92 Langfelder P, Horvath S. WGCNA: an R package for weighted correlation network analysis. BMC Bioinformatics. 2008;9:559.

93 Fortin JP, Triche TJ Jr, Hansen KD. Preprocessing, normalization and integration of the Illumina HumanMethylationEPIC array with minfi. Bioinformatics. 2017;33(4):55860.

94 Kudryashova KS, Burka K, Kulaga AY, Vorobyeva NS, Kennedy BK. Aging biomarkers: from functional tests to multi-omics approaches. Proteomics. 2020; 20(5-6): e1900408.
95 Blodgett JM, Theou O, Mitnitski A, Howlett SE, Rockwood K. Associations between a laboratory frailty index and adverse health outcomes across age and sex. Aging Med. 2019;2(1):11-7.

96 Horvath S. DNA methylation age of human tissues and cell types. Genome Biol. 2013; 14(10):R115.

97 Levine ME, Lu AT, Quach A, Chen BH, Assimes TL, Bandinelli S, et al. An epigenetic biomarker of aging for lifespan and healthspan. Aging. 2018;10(4):573-91.

98 Horvath S, Raj K. DNA methylation-based biomarkers and the epigenetic clock theory of ageing. Nat Rev Genet. 2018;19(6):37184.

99 Marioni RE, Suderman M, Chen BH, Horvath S, Bandinelli S, Morris T, et al. Tracking the epigenetic clock across the human life course: a meta-analysis of longitudinal cohort data. J Gerontol A Biol Sci Med Sci. 2019;74(1):5761

100 Alpert A, Pickman Y, Leipold M, Rosenberg-Hasson Y, Ji X, Gaujoux R, et al. A clinically meaningful metric of immune age derived from high-dimensional longitudinal monitoring. Nat Med. 2019;25(3):48795.

101 Franceschi C, Campisi J. Chronic inflammation (inflammaging) and its potential contribution to age-associated diseases. J Gerontol A Biol Sci Med Sci. 2014;69(Suppl 1):S4-9.

102 Franceschi C, Garagnani P, Parini P, Giuliani C, Santoro A. Inflammaging: a new immune-metabolic viewpoint for age-related diseases. Nat Rev Endocrinol. 2018; 14(10):576-90.

103 Lara J, Cooper R, Nissan J, Ginty AT, Khaw $\mathrm{KT}$, Deary IJ, et al. A proposed panel of biomarkers of healthy ageing. BMC Med. 2015; 13:222.

104 Purde M-T, Nock S, Risch L, Medina Escobar P, Grebhardt C, Nydegger UE, et al. The cystatin $\mathrm{C} /$ creatinine ratio, a marker of glomerular filtration quality: associated factors, reference intervals, and prediction of morbidity and mortality in healthy seniors. Translational Res. 2016;169:80 90.

105 Sebastiani P, Thyagarajan B, Sun F, Schupf $\mathrm{N}$, Newman AB, Montano $\mathrm{M}$, et al. Biomarker signatures of aging. Aging Cell. 2017;16(2):329-38

106 Klemera P, Doubal S. A new approach to the concept and computation of biological age. Mech Ageing Dev. 2006;127(3):240-8.

107 Levine ME. Modeling the rate of senescence: can estimated biological age predict mortality more accurately than chronological age? J Gerontol A Biol Sci Med Sci. 2013;68(6): $667-74$.

108 Liu Z, Kuo PL, Horvath S, Crimmins E, Ferrucci $L$, Levine $M$. A new aging measure captures morbidity and mortality risk across diverse subpopulations from NHANES IV: a cohort study. PLoS Med. 2018;15(12): e1002718.
Laboratory Assays as Biomarkers of Ageing
Gerontology 2021;67:503-516 DOI: $10.1159 / 000517659$ 
109 Ahadi S, Zhou W, Schüssler-Fiorenza Rose SM, Sailani MR, Contrepois K, Avina M, et al. Personal aging markers and ageotypes revealed by deep longitudinal profiling. Nat Med. 2020;26(1):83-90.

110 Rutenberg AD, Mitnitski AB, Farrell SG, Rockwood K. Unifying aging and frailty through complex dynamical networks. Exp Gerontol. 2018;107:126-9.

111 Lu AT, Quach A, Wilson JG, Reiner AP, Aviv A, Raj K, et al. DNA methylation GrimAge strongly predicts lifespan and healthspan. Aging. 2019;11(2):303-27.

112 Rezwan FI, Imboden M, Amaral AFS, Wielscher M, Jeong A, Triebner K, et al. Association of adult lung function with accelerated biological aging. Aging. 2020;12(1):518-42.

113 Lu AT, Seeboth A, Tsai PC, Sun D, Quach A, Reiner AP, et al. DNA methylation-based estimator of telomere length. Aging. 2019; 11(16):5895-923.

114 Lehallier B, Gate D, Schaum N, Nanasi T, Lee SE, Yousef $\mathrm{H}$, et al. Undulating changes in human plasma proteome profiles across the lifespan. Nat Med. 2019;25(12):1843-50.

115 Belsky DW, Moffitt TE, Cohen AA, Corcoran DL, Levine ME, Prinz JA, et al. Eleven telomere, epigenetic clock, and biomarkercomposite quantifications of biological aging: do they measure the same thing? Am J Epidemiol. 2018;187(6):1220-30.

116 Goldman DP, Cutler D, Rowe JW, Michaud PC, Sullivan J, Peneva D, et al. Substantial health and economic returns from delayed aging may warrant a new focus for medical research. Health Aff. 2013;32(10):1698-705.

117 Lopez-Otin C, Kroemer G. Hallmarks of Health. Cell. 2021;184(1):33-63.

118 Conti AA. Historical evolution of the cocept of health in Western medicine. Acta Biomed. 2018;83(3):352-4.

119 Morris BJ, Willcox BJ, Donlon TA. Genetic and epigenetic regulation of human aging and longevity. Biochim Biophys Acta Mol Basis Dis. 2019;1865(7):1718-44
120 Andreassen SN, Ben Ezra M, ScheibyeKnudsen M. A defined human aging phenome. Aging. 2019;11(15):5786-806.

121 Risch M, Sakem B, Risch L, Nydegger UE. The SENIORLAB study in the quest for healthy elderly patients. J Lab Med. 2018; 42(2):109-20.

122 Kuri-Morales P, Emberson J, Alegre-Díaz J, Tapia-Conyer R, Collins R, Peto R, et al. The prevalence of chronic diseases and major disease risk factors at different ages among 150,000 men and women living in Mexico City: cross-sectional analyses of a prospective study. BMC Public Health. 2009;9:9.

123 Solé-Auró A, Michaud PC, Hurd M, Crimmins E. Disease incidence and mortality among older Americans and Europeans. Demography. 2015;52:593-611.

124 Sydenstricker E. The incidence of various diseases according to age: Hagerstown morbidity studies no. VIII. Public Health Rec. 1928;43(19):1124-56.

125 Edvardsson M. Circulating levels and assessment of clinical laboratory analytes in $>80$ - year-old, apparently helathy, moderately healthy and frail individuals. Linköping, Sweden: Linköping University; 2019.

126 Salvioli S, Capri M, Bucci L, Lanni C, Racchi $\mathrm{M}$, Uberti $\mathrm{D}$, et al. Why do centenarians escape or postpone cancer? The role of IGF-1, inflammation and p53. Cancer Immunol Immunother. 2009;58(12):1909-17.

127 Sarkar TJ, Sebastiano V. Rejuvenation on the road to pluripotency, pluripotent sem cells - from the bench to the clinic. IntechOpen; 2016.

128 Goronzy JJ, Weyand CM. Mechanisms underlying $\mathrm{T}$ cell ageing. Nat Rev Immunol. 2019;19(9):573-83.
129 Zhu Y, Liu X, Ding X, Wang F, Geng X. Telomere and its role in the aging pathways: telomere shortening, cell senescence and mitochondria dysfunction. Biogerontology. 2019 Feb;20(1):1-16.

130 Dogra P, Rancan C, Ma W, Toth M, Senda $\mathrm{T}$, Carpenter DJ, et al. Tissue determinants of human NK cell development, function, and residence. Cell. 2020 Feb 20;180(4):749763.e13.

131 Petersen AM, Pedersen BK. The anti-inflammatory effect of exercise. J Appl Physiol. 2005 Apr;98(4):1154-62.

132 De Cecco M, Ito T, Petrashen AP, Elias AE, Skvir NJ, Criscione SW, et al. L1 drives IFN in senescent cells and promotes age-associated inflammation. Nature. 2019 Feb; 566(7742):73-78

133 Franceschi C, Garagnani P, Parini P, Giuliani C, Santoro A. Inflammaging: a new immune-metabolic viewpoint for age-related diseases. Nat Rev Endocrinol. 2018 Oct; 14(10):576-590.

134 Fülöp T, Larbi A, Witkowski JM. Human inflammaging. Gerontology. 2019;65(5):495504 .

135 Terman A, BrunkLipofuscin UT. mechanisms of formation and increase with age. APMIS. 1998 Feb;106(2):265-76.

136 Uchil PD, Nagarajan A, Kumar P. $\beta$-Galactosidase. Cold Spring Harb Protoc. 2017 Oct;32017(10):db.top096198.

137 Giordano A, Smorlesi A, Frontini A, Barbatelli G, CintiWhite S., brown and pink adipocytes: the extraordinary plasticity of the adipose organ. Eur J Endocrinol. 2014 170(5):R159-71.

138 Arques S. Human serum albumin in cardiovascular diseases. Eur J Intern Med. 2018 Jun;52:8-12.

139 Xiang Y, Wang SH, Wang L, Wang ZL, Yao $\mathrm{H}$, Chen LB, et al. Effects of ginsenoside Rg1 regulating Wnt/beta-catenin signaling on neural stem cells to delay brain senescence. Stem Cells Int. 2019 Dec;42019:5010184. 